\title{
Pensamiento crítico, resolución de problemas y comprensión lectora en ingresantes a la universidad
}

\author{
Critical thinking, problem solving and reading comprehension in college entrants
}

Luis Yarlequé Chocas 1

Leda Javier Alva ${ }^{2}$

Edith Nuñez LLacuachaqui 3

Linda Navarro García 4

Maribel Padilla Sánchez 5

RECIBIDO: 4 DE MARZO DE 2020

ACEPTADO: 11 DE JUNIO DE 2020

\section{RESUMEN}

Se trató de establecer sí, a un mayor nivel de desarrollo del pensamiento crítico corresponde también mayor capacidad para resolver problemas y mayor nivel de comprensión lectora en ingresantes a la universidad. Se empleó el método descriptivo, con diseño descriptivo correlacional. Se trabajó con 513 ingresantes a la Universidad Nacional del Centro del Perú, de ocho facultades, tomados por

\footnotetext{
Doctor en Psicología, Psicólogo, Docente, Universidad Nacional del Centro del Perú, lyarleque@uncp.edu.pe, ORCID: https://orcid.org/ 0000-0001-6028-578X),

Google Scholar: https://scholar.google.es/citations?user=wnwXAbOAAAAJ\&hl=es)

2 Doctora en Psicología, Psicóloga, Docente, Universidad Continental, ljavier@continental.edu.pe, ORCID: https://orcid.org/0000-0002-9793-1033,

Google Scholar: https://scholar.google.com/citations?user=ZgEVw6AAAAAJ\&hl=es\&oi=ao i

3 Doctora en Ciencias de la Educación, Educadora Docente, Universidad Nacional del Centro del Perú, enunez@uncp.edu.pe, ORCID: https://orcid.org/0000-0002-6219-00366,

Google Scholar: https://scholar.google.es/citations?view_op=list_works\&hl=es\&user=QglJBaYAAAAJ

4 Magister en Ciencias de la Comunicación, Comunicadora, Docente, Universidad Nacional del Centro del Perú, Inavarro@uncp.edu.pe, ORCID: https://orcid.org/0000-0003-0341-1422,

Google Scholar: https://scholar.google.com/citations?user=slgIDKsAAAAJ\&hl=es

5 Magister en Educación, Profesora, Docente, Universidad Nacional del Centro del Perú, mpadillas@uncp.edu.pe, ORCID: https://orcid.org/ 0000-0002-6492-0610,

Google Scholar: https://scholar.google.com/citations?user=Ymd4_YgAAAAJ\&hl=es\&authuser=1
}

Socialium, revista científica de Ciencias Sociales, Vol 4 - No. 2, julio - diciembre 2020, pág. 349-376. 


\section{0}

accesibilidad y consentimiento informado. Los instrumentos fueron desarrollados por los investigadores. Algunos resultados y conclusiones fueron, que el 35,09\% ingresan con bajo nivel de pensamiento crítico; que en las mujeres esta competencia está más desarrollada que en los varones; que el $57.89 \%$ de ingresantes tienen bajo nivel en la capacidad de resolución de problemas y que en eso no hay diferencias por sexo; el 33.528 se encuentra en el nivel bajo en comprensión lectora; que las mujeres también superan a los varones en ésta y, que el pensamiento crítico, está asociado con aquella significativamente según el coeficiente gamma de Goodman y Kruskal (Gamma $=0.189$ y P. valor $=0.00<0.05$ ). De ello, se desprende que, para ingresar a la universidad no es requisito indispensable tener desarrollado el pensamiento crítico, la capacidad para resolver problemas ni la comprensión lectora, lo cual acusa una seria limitación en el proceso de selección.

Palabras clave: Pensamiento crítico, resolución de problemas, comprensión lectora, sexo.

\section{ABSTRACT}

It was tried to establish if, to a higher level of development of critical thinking there is also a greater capacity to solve problems and a higher level of reading comprehension in university entrants. The descriptive method was used, with a correlational descriptive design. It worked with 513 entrants to the National University of Central Peru, from eight faculties, taken for accessibility and informed consent. The instruments were developed by the researchers. Some results and conclusions were that $35.09 \%$ enter with a low level of critical thinking; that in women this competence is more developed than in men; that $57.89 \%$ of entrants have a low level of problemsolving capacity and that there are no differences by sex; $33,52 \%$ is at the low level in reading comprehension; that women also outperform men in this and that critical thinking is associated with that significantly according to the Goodman and Kruskal gamma coefficient (Gamma $=0.189$ and P. value $=0.00<0.05$ ). From that, it follows that, to enter the university, it is not an essential requirement to have developed critical thinking, the ability to solve problems neither reading comprehension, which accuses a serious limitation in the selection process.

Keywords: Critical thinking, solving problems, reading comprehension, sex. 


\section{Introducción}

De acuerdo con los resultados de PISA (2019) reportados por la BBC News Mundo (2018) y el Ministerio de Educación (2016) los estudiantes peruanos tienen un bajo nivel de comprensión lectora. A este respecto, el MED reportó que sólo el $14.7 \%$ de estudiantes del segundo de secundaria, que fueron evaluados, leen de manera satisfactoria, mientras que el $23.7 \%$, cerca de medio millón de estudiantes, están en el nivel "previo al inicio"; lo que lleva a inferir que carecen de los aprendizajes necesarios para estar en dicho grado. Algo parecido ocurre con los estudiantes universitarios (Evaluación Censal de estudiantes, 2019. Las observaciones empíricas muestran que muchos de ellos tienen serias deficiencias al leer y por supuesto también en su comprensión de los textos y lo que es peor, no se han registrado diferencias entre los de primero y últimos ciclos (Mendoza, 2016). Este mismo fenómeno, se repite cuando se comparan profesores de los diferentes niveles educativos desarrollar (Yarlequé, Javier; Nuñez, Navarro, Cerrón y Monroe, 2012), los investigadores encontraron también, que el nivel educativo en que se labora, los estudios de posgrado, además de otras variables, parecen no marcar diferencias en la comprensión lectora de tal población; lo que conduce a presumir que esta variable no es tan fácil de desarrollar (Yarlequé et al., 2012).

Ahora bien, Cooper (1998) define la comprensión lectora como el proceso de elaborar el significado por la vía de aprender las ideas relevantes del texto y relacionarlas con las ideas que ya se tienen. La comprensión lectora se logra cuando el estudiante relaciona los nuevos conocimientos con los anteriormente adquiridos (Ausubel, 1983). En esa misma línea, años más tarde Manuale (2007) señala que la comprensión es un estado de capacitación para ejercitar determinadas actividades de comprensión como la explicación, ejemplificación, aplicación, justificación, comparación y contraste, contextualización y generalización.

Los teóricos (Solé, 2001) tienden a hablar de un nivel literal, que implica reproducir de una manera más o menos exacta los contenidos del texto. Un segundo nivel al que se ha dado por llamar inferencial que involucra procesos de hipotetización que permiten al individuo deducir el significado de las palabras, a partir del contexto, y por lo tanto reemplazar unas por otras así como prever el desenlace de la trama y un tercer nivel (el más elevado) al que se denomina crítico que pone en acción ya no solo la memoria y percepción sino que fundamentalmente requiere del concurso del pensamiento crítico; el cual permite que el lector tome posición respecto de los contenidos del texto. 


\section{2}

Por otro lado, la resolución de problemas es una forma del pensamiento (Luria, 1974) que se va logrando en el proceso de confrontación del individuo con situaciones problemáticas y pedagógicas (Gutiérrez, 2012).

Luria $(1974,2013)$, Schoenfeld (1985) y Carretero (1995) coinciden en que la resolución de problemas es una función del pensamiento en la que concurren varios fenómenos y procesos cognitivos y consiste en resolver una situación para la cual no existe una respuesta o solución en la memoria del individuo, no lo puede hacer manipulando, por ensayo y error, ni siquiera basta que comprenda el problema para que lo resuelva. De acuerdo con Luria (2013) resolver un problema, requiere en primera instancia, que el individuo se dé cuenta de que está frente a uno, que inhiba su tendencia natural a emitir respuestas espontáneas y se oriente en los datos del problema. Hecho esto, el individuo se plantea una serie de hipótesis de solución, de entre las cuales selecciona la más plausible y elige los medios para poner a prueba su hipótesis. Posteriormente, ejecuta su plan de solución y verifica sí el resultado satisface las condiciones del problema. Si es así el proceso termina, de no serlo se reinicia el proceso, poniendo a prueba otra de las hipótesis iniciales.

De otra parte, Faccione (2007), Rugarcía (1999) y Saiz y Rivas (2008) refieren que el pensamiento crítico es un proceso que tiene como fin el conocimiento a través del despliegue de habilidades de razonamiento, toma de decisiones, análisis de información que nos llevará a tener los resultados deseados. Así pues, el pensamiento crítico es una competencia, en tanto implica un componente procedimental, que es el que permite al individuo analizar, comprender, evaluar, interpretar, realizar inferencias y autorregular su conducta. Pero żcon qué opera? Con teorías, ideas o creencias que son el componente cognitivo. No obstante, ello supone una disposición mental del individuo, para hacer todo eso; esto es la actitud. En efecto, el pensamiento crítico supone someter a análisis y juicio aún las propias creencias y es precisamente esto lo que requiere la disposición del individuo para enjuiciar realmente todo.

La persona que tiene desarrollado el pensamiento crítico, razona de modo que, puede descubrir las falacias y las conclusiones que no tienen sustento, busca información, se formula preguntas acerca de los hechos que le preocupa, analizan los conceptos, las premisas y los puntos de vista, ajenos, pero también los suyos. Es capaz, de cambiar de enfoque al examinar los hechos. Si se percata de que hubo error en lo que suscribió antes, puede cambiar su posición al respecto. Hace metacognición, es decir es consciente de 
su propio proceso de pensar y finalmente, puede supeditar sus emociones y sentimientos a la razón (Campos, 2007).

Claro está, que los fenómenos y procesos psíquicos se desarrollan de lo simple a lo complejo. Así el desarrollo de un fenómeno psíquico superior, se apoya en sus formas más simples y a su vez el desarrollo del fenómeno superior estimula a los que les subyacen; en una dialéctica permanente.

Sobre pensamiento crítico, han investigado Pineda y Cerrón (2015) quienes estudiaron la relación del pensamiento crítico y rendimiento académico en universitarios; por su parte, Guevara (2016) investigó acerca de la relación del desempeño docente y el pensamiento crítico en estudiantes del décimo ciclo; Macedo (2018) investigó acerca del pensamiento crítico y el rendimiento académico en ingresantes a la Universidad Nacional de Ingeniería, también se han ocupado del tema Alejo (2017); Bringas, Caro y Trigozo (2018), entre otros.

Entorno a la resolución de problemas se han hecho también una serie de trabajos como los de Javier (2003) quien comparó la estabilidad de la atención y la solución de problemas racionales en universitarios de Huancayo y Huancavelica. Pino (2012) estudió las concepciones y prácticas de resolución de problemas en estudiantes universitarios; también han estudiado el tema Mejía y Loangov (2014); Cruz (2015); Escalante (2015), entre otros. En cuanto a la comprensión lectora, han realizado algunos trabajos Yarlequé et al. (2012) quienes analizaron la comprensión lectora en docentes de la región Junín. A partir de los resultados, los autores Yarlequé, Nuñez y Navarro (2012) experimentaron un programa para mejorar la lectura en docentes de la región Junín. Por otro lado, Zárate (2019) estudió la autoeficacia, comprensión de lectura y rendimiento académico en ingresantes a una universidad privada en Lima. También han abordado el tema autores como Vela (2015); Alonso, Coronel y Guevara (2016); Cuñachi y Leiva (2018).

No obstante, en todas estas investigaciones, no se ha estudiado la relación entre pensamiento crítico, capacidad para resolver problemas y comprensión lectora, por ello en el presente estudio, se propuso resolver el siguiente problema. ¿́Los ingresantes a la universidad con mayor desarrollo del pensamiento crítico tienen también mayor capacidad para resolver problemas y mayor nivel de comprensión lectora que sus análogos con bajo nivel de pensamiento crítico? En concordancia con ello, el objetivo buscaba establecer sí, a un mayor nivel de desarrollo del pensamiento crítico corresponde también mayor capacidad para resolver problemas y mayor nivel de comprensión lectora en ingresantes a la universidad. La hipótesis proponía que: Los 


\section{4}

ingresantes a la universidad con mayor desarrollo del pensamiento crítico tienen también mayor capacidad para resolver problemas y mayor nivel de comprensión lectora que sus análogos con bajo nivel de pensamiento crítico.

Esta investigación se justifica en el hecho de que, pese a que se ha realizado una serie de estudios, acerca del pensamiento crítico, la resolución de problemas y la comprensión lectora - algunos de los cuales se cita líneas arriba - no se encontraron estudios que hayan abordado la relación de estas tres variables, lo cual significa que hay un vacío en el conocimiento, que es menester abordar. Los resultados de este estudio tienen implicancias teóricas y prácticas. Teóricas, en primera instancia, porque ha permitido acercarnos al análisis de la relación entre pensamiento crítico y resolución de problemas y poner de manifiesto una incongruencia; en la supuesta confluencia de procesos cognitivos entre ambas variables lo cual obliga a seguir investigando al respecto. En segundo lugar, porque ha ratificado la hipótesis de que a un mayor desarrollo de pensamiento crítico corresponde un mayor desarrollo de la comprensión lectora. Lo cual es muy importante, porque ratifica los supuestos teóricos de la relación entre ambas variables.

En el terreno práctico, el trabajo brinda valiosa información a los docentes de todos los niveles educativos por lo menos de la región Junín del Perú, pero además proporciona una idea de lo que debe estar ocurriendo en el resto del país, al respecto de las variables aquí estudiadas. Finalmente, la información referida podrá ser empleada por la Dirección Regional de Educación de Junín (DREJ) y por el Ministerio de Educación Peruano (MED) para redireccionar sus políticas poniendo el énfasis debido en el desarrollo de estas variables en el estudiante peruano. Pero también por las sucesivas comisiones de admisión de la Universidad Nacional del Centro del Perú y probablemente por otras universidades que deseen mejorar su proceso de admisión, no solo en la región sino también en el país, ya que es muy posible que los resultados aquí expuestos no difieran mucho de los de otras regiones del Perú.

\section{Método}

La investigación que se presenta es de tipo sustantiva (Sánchez y Reyes, 2016). Se empleó el método descriptivo, con diseño descriptivo correlacional (Alarcón, 1991; Sánchez y Reyes, 2016; Yarlequé, Javier, Monroe y Nuñez, 2007). 


\section{Población y muestra}

La población estuvo constituida por los ingresantes a la Universidad Nacional del Centro del Perú en el 2018, que es la Universidad más importante de la región central del país, la única de Huancayo en la que el examen de admisión, tiene un carácter selectivo. Regularmente en el examen más grande suelen competir un promedio de 6 mil postulantes procedentes de diferentes instituciones educativas públicas y privadas, grandes y pequeñas, de la región, y de regiones vecinas. De ahí que sus postulantes e ingresantes constituyen una muestra representativa de los estudiantes de la región central del Perú y no solo de la región Junín. Se tomó una muestra intencional, de 513 estudiantes tomando en cuenta: la accesibilidad y el consentimiento informado. Los estudiantes eran informados de lo que se trataba en la investigación y se les daba la opción de responder las pruebas si así lo querían. Solo se tomaron en cuenta en la muestra a los que habían desarrollado las tres pruebas. Así, se obtuvo una muestra final de ingresantes a las carreras de: administración, antropología, ciencias de la comunicación, contabilidad, enfermería, ingeniería civil, ingeniería mecánica y trabajo social; lo cual constituye el $30 \%$ de las carreras que se brindan en la universidad. De los 513 estudiantes, 244 fueron mujeres y 269 varones cuyas edades oscilaban aproximadamente entre 17 y 24 años.

\section{Las técnicas e instrumentos de investigación}

Las técnicas empleadas fueron la observación indirecta no reactiva, debido a que, ninguna de las tres variables en estudio, pueden ser observadas directamente, sino que se observan las respuestas de los individuos frente a los estímulos y estas, las respuestas constituyen una expresión de los procesos físicos subyacentes que no son directamente observables. La encuesta se utilizó para aplicar los instrumentos a la muestra.

Se utilizó la prueba de pensamiento crítico desarrollada por los investigadores la cual estuvo constituida por seis ítems, los cinco primeros requerían que el estudiante razonara acerca del contenido y lógica de los enunciados que se les proporcionaban y que dé su opinión en los cuatro primeros casos. El quinto contenía una creencia muy difundida en diversos estratos de la sociedad peruana. Los examinados debieron analizar los enunciados e interpretarlos en primera instancia, lvego manifestar su acuerdo o su desacuerdo con el enunciado y fundamentar su respuesta. El quinto ítem además de eso exigió que el examinado haga una inferencia respecto del por qué de la creencia, señalando su acuerdo o su desacuerdo, lo justifique y además que autoevalúe su 


\section{6}

disposición al cambio, la misma que también debió ser fundamentada. El sexto ítem consistió en un problema, el cual exigió para ser resuelto, analizar y contrastar las versiones de algunos personajes e inferir que proposiciones fueron verdaderas y cuales falsas. El ítem no solo requiere contestar a la pregunta sino también fundamentarla. En toda la prueba, el examinado puede obtener puntajes de 0 a 18. La prueba permite identificar tres niveles del pensamiento crítico: bajo, medio y alto cuyos valores se establecieron estadísticamente tomando los percentiles 33, 67 y 100.

La prueba posee confiabilidad de consistencia interna, establecida mediante Alfa de Cronbach igual a 0,704. El análisis de la validez de los ítems, se estableció con la misma prueba obteniéndose un coeficiente total de 0.312 , mayor a 0.30 que es lo esperado.

La batería para evaluar resolución de problemas, fue desarrollada con base en los trabajos de Yarlequé (1994), Matalinares (1999) y Javier (2004) y está constituida por tres problemas, dos cerrados y uno abierto, el segundo problema cerrado que se presenta al examinado tiene los mismos fundamentos lógicos que el primero, aunque con datos y situaciones distintas, lo cual permite examinar la resolución analógica de problemas. El tercer problema es de tipo abierto. Por tanto, no hay una respuesta posible sino varias. En este ítem obtiene el mayor puntaje el que puede dar la respuesta más eficiente. La prueba posee validez de contenido con un coeficiente de concordancia de Kendall de 0.85 reportada por Javier (2004), la confiabilidad se obtuvo mediante el alfa de Cronbach cuyo coeficiente es 0,649.

La prueba de comprensión lectora fue desarrollada (validada y confiabililizada para efectos de la investigación) consta de un texto que se propone a los examinados luego de lo cual deben responder a 11 ítems (seis cerrados, uno semi abierto y cuatro abiertos) que permiten discriminar los tres niveles de comprensión lectora: literal, inferencial y crítico, como también los procesos de la memoria, percepción y pensamiento.

Esta prueba posee coeficiente de estabilidad de $0.023<0.05$ hallado mediante la técnica de test retes, lo cual indica que la prueba es confiable. Así mismo, se halló la consistencia interna de la prueba mediante Alfa de Cronbach, la misma que asciende a 0.757, cuyo coeficiente de correlación corregido es de 0.408 que indica la validez de los ítems (Fátima, 2012). Los ítems 4,6, 7 y 10 obtuvieron puntuaciones cercanas a 0.35 indicado Fátima, (2012), Sin embargo dada la importancia de lo que exploran los ítems, los autores decidieron que dichos ítems forman parte del instrumento. 


\section{Resultados}

\section{Tabla 1}

Niveles de Pensamiento Crítico en la muestra

\begin{tabular}{lcc}
\hline \hline Nivel & $\mathrm{Fi}$ & $\mathrm{Hi}$ \\
\hline Bajo & 180 & 35,1 \\
Medio & 226 & 44,1 \\
Alto & 107 & 20,9 \\
Total & 513 & 100 \\
\hline \hline
\end{tabular}

\section{Tabla 2}

Niveles de Resolución de Problemas en la muestra

\begin{tabular}{lll}
\hline \hline Niveles & $\mathrm{Fi}$ & $\mathrm{Hi}$ \\
\hline Bajo & 297 & 57,9 \\
Medio & 97 & 18,9 \\
Alto & 119 & 23,2 \\
Total & 513 & 100.0 \\
\hline \hline
\end{tabular}

\section{Tabla 3}

Niveles de Comprensión Lectora en la muestra

\begin{tabular}{lll}
\hline \hline Niveles & $\mathrm{Fi}$ & $\mathrm{Hi}$ \\
\hline Bajo & 172 & 33,5 \\
Medio & 202 & 39,4 \\
Alto & 139 & 27,1 \\
Total & 513 & 100.0 \\
\hline \hline
\end{tabular}

\section{Tabla 4}

Pensamiento crítico de acuerdo al sexo en la muestra

\begin{tabular}{llll}
\hline \hline & Valor & Gl & $\begin{array}{l}\text { Significación } \\
\text { asintótica }\end{array}$ \\
\hline $\begin{array}{l}\text { Chi-cuadrado de } \\
\begin{array}{l}\text { Pearson } \\
\text { Razón de }\end{array}\end{array}$ & $21,864^{a}$ & 2 & .000 \\
$\begin{array}{l}\text { verosimilitud } \\
N \text { de casos válidos }\end{array}$ & 22.138 & 2 & .000 \\
\hline \hline
\end{tabular}




\section{Tabla 5}

Pensamiento Crítico y tipo de gestión Educativa: Estatal y particular

\begin{tabular}{lccc}
\hline \hline & Valor & Gl & $\begin{array}{c}\text { Significación } \\
\text { asintótica } \\
\text { (bilateral) }\end{array}$ \\
\hline $\begin{array}{l}\text { Chi-cuadrado de } \\
\text { Pearson }\end{array}$ & 15,170 a & 2 & .001 \\
Razón de verosimilitud & 15.121 & 2 & .001 \\
N de casos válidos & 513 & & \\
\hline \hline
\end{tabular}

\section{Tabla 6}

Comparación del pensamiento crítico de acuerdo a los años de egreso de la secundaria

\begin{tabular}{lccc}
\hline \hline & Valor & Gl & $\begin{array}{c}\text { Significación } \\
\text { asintótica }\end{array}$ \\
\hline $\begin{array}{l}\text { Chi-cuadrado de } \\
\text { Pearson }\end{array}$ & $1,578^{a}$ & 6 & .954 \\
Razón de verosimilitud & 1.572 & 6 & .955 \\
No de casos $^{\circ}$ & 513 & & \\
\hline \hline
\end{tabular}

\section{Tabla 7}

Pensamiento crítico y el número de Intentos de ingreso a la universidad

\begin{tabular}{lccc}
\hline \hline & Valor & Gl & $\begin{array}{c}\text { Significación } \\
\text { asintótica }\end{array}$ \\
\hline Chi-cuadrado de Pearson & $12,309 a$ & 6 &, 055 \\
Razón de verosimilitud & 12,212 & 6 &, 057 \\
$N^{\circ}$ de casos & 513 & & \\
\hline
\end{tabular}

\section{Tabla 8}

Comparación del pensamiento crítico de acuerdo a estudios paralelos

\begin{tabular}{lccc}
\hline \hline & Valor & Gl & $\begin{array}{c}\text { Significación } \\
\text { asintótica }\end{array}$ \\
\hline $\begin{array}{l}\text { Chi-cuadrado } \\
\begin{array}{l}\text { de Pearson } \\
\text { Razón }\end{array}\end{array}$ & $.029 a$ & 2 & .985 \\
$\begin{array}{l}\text { verosimilitud } \\
N^{\circ} \text { de casos }\end{array}$ & .029 & 2 & .985 \\
\hline \hline
\end{tabular}




\section{Tabla 9}

Comparación de la capacidad para resolver problema de acuerdo al sexo

\begin{tabular}{lccc}
\hline \hline & Valor & Gl & $\begin{array}{c}\text { Significación } \\
\text { asintótica (bilateral) }\end{array}$ \\
\hline $\begin{array}{l}\text { Chi-cuadrado de } \\
\text { Pearson }\end{array}$ & $2,329 a$ & 2 & .312 \\
Razón de & 2.337 & 2 & .311 \\
verosimilitud & & & \\
N de casos válidos & 513 & & \\
\hline \hline
\end{tabular}

\section{Tabla 10}

Capacidad para resolver problemas de acuerdo al número de intentos de para ingresar a la universidad

\begin{tabular}{lccc}
\hline \hline & Valor & Gl & $\begin{array}{l}\text { Significación } \\
\text { asintótica }\end{array}$ \\
\hline Chi-cuadrado de Pearson & $4,455^{a}$ & 6 & .615 \\
Razón de verosimilitud & 4.335 & 6 & .631 \\
$N$ de casos válidos & 513 & & \\
\hline \hline
\end{tabular}

\section{Tabla 11}

Resolución de problemas de acuerdo de gestión de la Institución Educativa de procedencia

\begin{tabular}{llcc}
\hline \hline & Valor & Gl & $\begin{array}{c}\text { Significación } \\
\text { asintótica }\end{array}$ \\
\hline $\begin{array}{l}\text { Chi-cuadrado de } \\
\text { Pearson }\end{array}$ & $4,767 a$ & 2 & .092 \\
Razón de verosimilitud & 4.885 & 2 & .087 \\
N de casos & 513 & & \\
\hline \hline
\end{tabular}

\section{Tabla 12}

Resolución de problemas según los años de egreso de la secundaria

\begin{tabular}{ccc}
\hline \hline Valor & Gl & Significación asintótica \\
\hline $10,149 a$ & 6 & .119 \\
10.525 & 6 & .104 \\
513 & & \\
\hline \hline
\end{tabular}

\section{Tabla 13}




\section{0}

Capacidad de resolución de problemas de acuerdo a estudios paralelos en la universidad

\begin{tabular}{lcc}
\hline \hline Valor & Gl & $\begin{array}{c}\text { Significación asintótica } \\
\text { (bilateral) }\end{array}$ \\
\hline 3,586 a & 2 & .166 \\
3.527 & 2 & .171 \\
513 & & \\
\hline \hline
\end{tabular}

\section{Tabla 14}

Comprensión lectora de acuerdo al sexo

\begin{tabular}{llcc}
\hline \hline & Valor & Gl & $\begin{array}{c}\text { Significación } \\
\text { asintótica (bilateral) }\end{array}$ \\
\hline $\begin{array}{l}\text { Chi-cuadrado de } \\
\text { Pearson }\end{array}$ & $9,410^{a}$ & 2 & .009 \\
Razón de verosimilitud & 9.445 & 2 & .009 \\
N de casos válidos & 513 & & \\
\hline \hline
\end{tabular}

\section{Tabla 15}

Niveles de comprensión lectora de acuerdo a la institución educativa de procedencia

\begin{tabular}{lcc}
\hline \hline Valor & Gl & $\begin{array}{c}\text { Significación asintótica } \\
\text { (bilateral) }\end{array}$ \\
\hline $1,297 a$ & 2 & .523 \\
1.299 & 2 & .522 \\
513 & & \\
\hline \hline
\end{tabular}

\section{Tabla 16}

Comprensión lectora de acuerdo a la cantidad de años de egresos de la secundaria

\begin{tabular}{ccc}
\hline \hline Valor & Gl & $\begin{array}{c}\text { Significación asintótica } \\
\text { (bilateral) }\end{array}$ \\
\hline $8,199 a$ & 6 & .224 \\
8.293 & 6 & .217 \\
513 & & \\
\hline \hline
\end{tabular}




\section{Tabla 17}

Comprensión lectora de acuerdo al número de intentos de ingresos en la universidad

\begin{tabular}{ccc}
\hline \hline Valor & Gl & $\begin{array}{c}\text { Significación } \\
\text { asintótica }\end{array}$ \\
\hline $6,907 a$ & 6 & .330 \\
6.808 & 6 & .339 \\
513 & & \\
\hline \hline
\end{tabular}

\section{Tabla 18}

Comprensión lectora y estudios paralelos a la universidad.

\begin{tabular}{ccc}
\hline \hline Valor & Gl & $\begin{array}{c}\text { Significación asintótica } \\
\text { (bilateral) }\end{array}$ \\
\hline .971 a & 2 & .615 \\
.966 & 2 & .617 \\
513 & & \\
\hline \hline
\end{tabular}

\section{Tabla 19}

Relación entre Pensamiento crítico y Comprensión lectora

\begin{tabular}{lccc}
\hline \hline & Valor & Gl & $\begin{array}{c}\text { Significación asintótica } \\
\text { (bilateral) }\end{array}$ \\
\hline $\begin{array}{l}\text { Chi-cuadrado de } \\
\text { Pearson }\end{array}$ & $18,553 a$ & 4 & .001 \\
Razón de verosimilitud & 18,376 & 4 & .001 \\
N de casos válidos & 513 & \\
\hline \hline
\end{tabular}

\section{Tabla 20}

Tabla Cruzada entre Pensamiento crítico y Comprensión lectora

\begin{tabular}{|c|c|c|c|c|c|c|}
\hline & & & \multicolumn{3}{|c|}{ nivel de pensamiento crítico } & \multirow[b]{2}{*}{ Total } \\
\hline & & & nivel bajo & $\begin{array}{c}\text { nivel } \\
\text { medio }\end{array}$ & nivel alto & \\
\hline \multirow{4}{*}{$\begin{array}{l}\text { nivel de } \\
\text { comprensión } \\
\text { lectora }\end{array}$} & \multirow{2}{*}{$\begin{array}{l}\text { nivel } \\
\text { bajo }\end{array}$} & Recuento & 76 & 68 & 28 & 172 \\
\hline & & $\begin{array}{l}\text { \% de } \\
\text { comprensión } \\
\text { lectora }\end{array}$ & $44.19 \%$ & $39.53 \%$ & $16.28 \%$ & $100.00 \%$ \\
\hline & \multirow{2}{*}{$\begin{array}{l}\text { nivel } \\
\text { medio }\end{array}$} & Recuento & 70 & 95 & 37 & 202 \\
\hline & & $\begin{array}{l}\text { \% de } \\
\text { comprensión } \\
\text { lectora }\end{array}$ & $34.65 \%$ & $47.03 \%$ & $18.32 \%$ & $100.00 \%$ \\
\hline
\end{tabular}




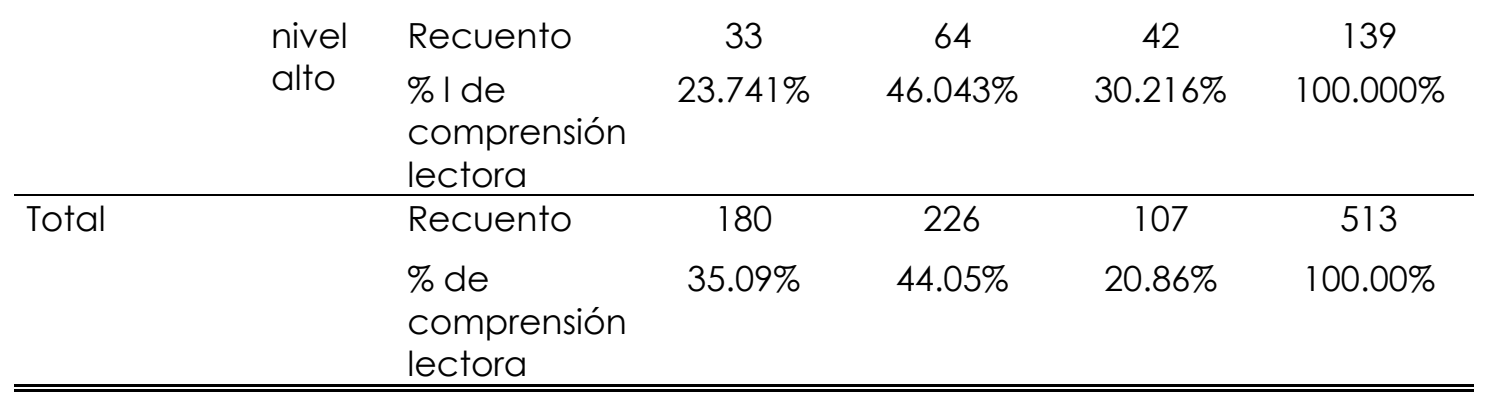

\section{Tabla 21}

Relación entre Pensamiento crítico y solución de problemas

\begin{tabular}{lccc}
\hline \hline & & & \\
& Valor & Gl & Significación asintótica (bilateral) \\
\hline Chi-cuadrado de Pearson & 2,258 a & 4 & .688 \\
Razón de verosimilitud & 2,327 & 4 & .676 \\
N de casos válidos & 513 & & \\
\hline \hline
\end{tabular}

\section{Tabla 22}

Relación entre Pensamiento crítico y Comprensión lectora.

\begin{tabular}{lccc}
\hline \hline & Valor & gl & $\begin{array}{c}\text { Significación } \\
\text { asintótica } \\
\text { (bilateral) }\end{array}$ \\
\hline $\begin{array}{l}\text { Chi-cuadrado } \\
\text { de Pearson }\end{array}$ & $18,553 a$ & 4 & .001 \\
$\begin{array}{l}\text { Razón de } \\
\text { verosimilitud }\end{array}$ & 18.376 & 4 & .001 \\
$\begin{array}{l}\text { N de casos } \\
\text { válidos }\end{array}$ & 513 & & \\
\hline \hline
\end{tabular}

\section{Discusión}

Los resultados mostraron que, del total de la muestra, 35 de cada 100, es decir más de la tercera parte se ubican en el nivel bajo de pensamiento crítico y sólo cerca de 21 de cada 100 presentarían altos niveles en esta forma del pensamiento, esto significa que únicamente la quinta parte de los jóvenes que ingresan a la universidad lo harían con alto nivel de pensamiento crítico. Nuestros hallazgos resultan sumamente interesantes, en primer lugar, porque en los documentos oficiales emanados por el Ministerio de Educación peruano: Diseño Curricular Nacional, Rutas de aprendizaje y mapas de 
progreso, que fueron los que guiaron la formación de estos estudiantes en las instituciones educativas, se hablaba ya del énfasis que se debería poner en su desarrollo. Los datos de que disponemos muestran que no se habría logrado por lo menos en una buena parte de quienes ingresaron a la universidad más exigente de la región central del Perú. Pero también mostrarían que la universidad no estaría evaluando esta competencia y ella no sería un requisito para ser estudiante universitario. Falta saber sí a lo largo de los estudios universitarios se logra su desarrollo ya que el trabajo de Batista (2008) mostraría que por lo menos los estudiantes de educación si pueden lograrlo con la influencia de sus docentes (citado por Gonzales, 2016).

Pero en términos cualitativos ¿̇qué significa que sólo dos de cada 10 tengan alto nivel de desarrollo en el pensamiento crítico? Implica que únicamente esta es aproximadamente - la proporción de la población de ingresantes que evalúan la validez de los razonamientos y basan su comportamiento en la comprensión de los conceptos (Rojas, citado por Altuve, 2010). Esto se pone de manifiesto cuando los estudiantes no aceptan el razonamiento de otros sin previamente analizar su consistencia lógica. En la prueba que enfrentaron, entre otras cosas se les daba la ocasión de analizar la lógica de una proposición tan difundida de que las personas tienen gripe debido al frío y que, para no enfermarse, hay que abrigarse. Cerca del 35\% de los jóvenes están dispuestos a aceptar este tipo de proposiciones como verdaderas pese a que sus estudios de biología les permiten conocer que la gripe está producida por virus. Esto les hace permeables a los razonamientos falaces debido a que no son capaces de someterlos a análisis crítico. Otra cosa de lo que también carecería por lo menos el 35\% de los jóvenes que ingresan a la universidad es de la actitud para analizar la información que reciben. Esto los hace proclives a recibir y aceptar información que puede no ser verdadera e incluso ilógica. Los resultados mostrarían también que solo 21 de cada 100 jóvenes examinan los contextos en que ocurren los hechos y están dispuestos a poner a prueba las teorías, aplicándolas en la práctica.

Al comparar mujeres con varones en pensamiento crítico, se encontró entre otras cosas que, cerca del doble de varones se hallan en el nivel bajo $(25,81 \%$ de las mujeres contra $43,49 \%$ de los varones) y en general hay ventaja de las mujeres en esta variable (verificado con Chi cuadrado. Ya que lo contrario ocurre en el nivel alto en el que se ubica el 27,45\% de las mujeres contra solo el 14,86\% de los varones. Es decir, por cada dos mujeres con alto nivel del pensamiento crítico habría únicamente un varón. Esto 


\section{4}

quiere decir que, las mujeres tendrían mayor desarrollo del pensamiento crítico que los varones por lo menos al ingresar a la universidad. Pero, ¿cómo se explica este fenómeno? En primer término, hay que reconocer que, el pensamiento crítico constituye una competencia que se desarrolla en el contexto de la educación formal e informal. Ya que no solo se trata de analizar, evaluar, someter a prueba las teorías, concepciones o proposiciones (Faccione, 2007) se trata también de la disposición mental para hacerlo, es decir de la actitud (Yarlequé y Moya, 2009; Sabucedo y Morales, 2015).

Ahora bien, el hecho de que la muestra incluya varones y mujeres de los mismos grados, niveles e instituciones educativas conduce a pensar que no sería la educación formal la que estaría marcando la diferencia sino más bien la informal, es decir aquella que se da en los hogares y en la sociedad en general, fuera de las instituciones educativas. Se hace necesario pues realizar otros estudios para dilucidar este aspecto. Ya que no se conoce la existencia de trabajos que lo hayan hecho. Por el momento solo estamos en condiciones de señalar que al parecer la educación que recibe la mujer en el contexto de la región central del Perú le brinda mejores condiciones para el desarrollo de su pensamiento crítico.

Cuando se comparó a los ingresantes a la universidad que proceden de instituciones educativas estatales con los que proceden de instituciones particulares, se encontró que habían también diferencias significativas, pero tales diferencias parecen explicarse no tanto porque hayan más estudiantes de instituciones estatales con nivel alto de pensamiento crítico sino más bien porque hay mucho más estudiantes de instituciones educativas particulares con bajo nivel de desarrollo del pensamiento crítico que los de las estatales y también hay más estudiantes de estos últimos en el nivel medio que de los particulares. Más bien, en el nivel alto son técnicamente similares ambos subgrupos. De esto surgen algunas interrogantes: ¿̇es la institución estatal la principal responsable de las diferencias apuntadas? estudiantes que asisten a instituciones estatales mejores para el desarrollo del pensamiento crítico que las que tienen los estudiantes de instituciones particulares? 0 son todo este conjunto de variables los responsables de tales diferencias. Existe entonces la necesidad de seguir investigando al respecto.

Otra es la situación cuando se compara a los estudiantes en función de la cantidad de años que hay entre el egreso de la secundaria y el ingreso a la universidad. En este caso el hecho de que el estudiante demore más o menos años en ingresar a la universidad parece ser una variable írrita respecto del desarrollo de su pensamiento crítico y algo 
análogo ocurre cuando se los compara en función del número de intentos para lograr su ingreso, lo que significaría que el hecho de que un estudiante haga más intentos que otro no le da mayor desarrollo del pensamiento crítico ni viceversa. Ahora bien, teniéndose en cuenta que, los estudiantes se prepararan en las academias para postular a la universidad, nuestros hallazgos sugieren que la preparación que reciben en aquellas no involucra el desarrollo de esta competencia, lo cual vuelve a poner en el tapete el hecho de que la universidad más importante de la región central del Perú no tiene como requisito para el ingreso a sus carreras, la referida competencia y es posible que esto mismo esté ocurriendo en muchas más universidades del Perú.

Algo similar ocurre cuando se compara a los que estudian únicamente la carrera a la que ingresaron, con los que estudian dos carreras paralelamente. Los resultados muestran que no hay diferencias entre ambos subgrupos, lo cual implicaría que estudie o no otra carrera, no influiría ni a favor ni en contra del pensamiento crítico, también puede colegirse razonablemente que al parecer la mayoría de las carreras no requeriría el desarrollo del pensamiento crítico. Dado que, la muestra con la que se ha trabajado incluía estudiantes de ciencias de la salud, ingenierías, ciencias sociales y de la empresa. Al examinar los resultados en la prueba de resolución de problemas se encontró que, el $57,9 \%$ de la muestra se encuentra en el nivel bajo. Es decir, más de la mitad de los ingresantes a la universidad que más exige para su ingreso en la región central del Perú, puede hacerlo sin tener desarrollada la capacidad para resolver problemas, lo que indicaría que tampoco esta capacidad se estaría evaluando en el examen de admisión y obliga a un estudio que pueda dilucidar si dicha capacidad se desarrolla a lo largo de la carrera. De otro lado, aunque no es posible generalizar nuestros resultados al Perú, puede suponerse que esto mismo esté ocurriendo en varias regiones sobre todo de las universidades estatales de las provincias peruanas que son las que tienen un examen de admisión cuyo fin es seleccionar a los mejores. No obstante, es necesario seguir investigando al respecto. Esto también vuelve a poner en discusión las bondades de la educación secundaria que se brinda a los estudiantes peruanos por lo menos en la mayoría de instituciones estatales y particulares. Ya que revelaría en primer término, que se puede terminar "exitosamente" la secundaria e ingresar a la universidad sin tener desarrollado el pensamiento crítico ni la capacidad de resolución de problemas, que también es una manifestación del pensamiento (Luria, 2013) ¿Qué están evaluando pues los exámenes de admisión? 


\section{6}

Nuestros hallazgos son tanto más reveladores, cuanto que se ha registrado solo el $23 \%$ en el nivel alto de la capacidad de resolución de problemas, es decir 23 de cada 100 ingresantes a la universidad enfrentan con éxito problemas abiertos y cerrados.

Por otra parte, no se registraron diferencias significativas entre la capacidad de resolver problemas en varones y mujeres, lo que quiere decir que independientemente de que se trate de varones o mujeres la mayoría de los jóvenes presenta las características apuntadas. Resultados análogos fueron reportados por Javier (2003). Pero ¿̇qué implica que los ingresantes a la universidad tengan en su mayoría un nivel bajo en la capacidad de resolución de problemas y que, solo el $23 \%$ entre varones y mujeres se hallan en el nivel alto? Significa que solo 23 de cada 100 de los jóvenes son capaces de inhibir la emisión de respuestas espontaneas frente a un problema, es decir de evitar proceder por ensayo y error, mientras que la mayoría no lo haría así, solo estos, analizarían la situación problemática, a partir de la orientación en los datos del problema y formularían una o más hipótesis que luego someten a prueba, cuyo resultado es finalmente evaluado para comprobar si la solución hallada satisface o no los requerimientos del problema (Luria, 1974, 1984). En cambio, más de la mitad no pasa por todas estas etapas y solo cumplirían algunas de ellas en el mejor de los casos, con lo cual reduce ostensiblemente su eficiencia para resolver problemas. Se encontró también que el número de intentos de ingresos a la universidad no mejora la capacidad para resolver problemas, lo que significa que ni el estudiante ni las academias que los preparan ponen énfasis en la referida capacidad, probablemente porque la consideran innecesaria para el logro de sus fines.

De otra parte, al comparar a los estudiantes en función de si proceden de instituciones educativas particulares o estatales, no se hallaron diferencias estadísticas entre ambos sub grupos hecho que, parece indicar que ni las instituciones particulares ni las estatales, ponen el suficiente énfasis en esta capacidad como para que se noten diferencias. Así pues, se completa el cuadro: los colegios, las academias, ni la universidad parecen dar la suficiente importancia a la capacidad de resolución de problemas en los estudiantes y si esta no se logra durante el trascurso de los estudios universitarios, entonces estaríamos frente a un panorama verdaderamente alarmante.

Al comparar la capacidad de resolver problemas en los estudiantes en función de los años trascurridos desde que terminaron la secundaria, no se hallaron diferencias significativas entre los subgrupos, ya que todos siguen la misma tendencia: presentan la mayor frecuencia en el nivel bajo, disminuye un poco en el nivel medio y la más baja 
frecuencia se registra en el nivel alto. En otras palabras, no importa cuántos años trascurra desde el egreso de la secundaria, un año o más de cuatro todos parecen seguir el mismo patrón lo cual sugiere que, por lo menos en este aspecto los ingresantes a la universidad no acusarían cambios.

En esta misma dirección apuntan los resultados respecto de los estudios paralelos. También en este caso el hecho de que los estudiantes sigan o no otras carreras no mejoran su capacidad para resolver problemas lo cual legítimamente permite pensar que estas otras carreras tampoco requieren dicha capacidad para que el estudiante responda con relativo "éxito". En este punto, es pertinente tomar en cuenta que la batería de problemas a la que fue enfrentada la muestra incluía dos problemas de los cuales el segundo podía ser resuelto por lo que algunos autores, han denominado resolución analógica (Polya, 1965), en otras palabras, la resolución del primero, podría por analogía, facilitar la resolución del segundo; no obstante, como se ha hecho notar, la mayoría de los jóvenes no lo logró. Aun cuando estos dos problemas eran de tipo cerrado (Garret, 1988) lo cual podría haber facilitado al estudiante su solución empleando tablas lógicas. El tercer problema que fue presentado es abierto. En él, el estudiante debía buscar la respuesta que mejor cumpla las condiciones exigidas, esto obliga a poner en acción el pensamiento divergente lateral (De Bono, 2014) y exige una evaluación de diversas opciones.

Veamos que se encontró en lo concerniente a la variable comprensión lectora. Allí del $100 \%$ de la muestra el $34 \%$ se halló en el nivel bajo, el $39 \%$ en el nivel medio y el $27 \%$ en el nivel alto. Es decir 34 de cada 100 jóvenes (más de la tercera parte de la muestra) tiene dificultades para comprender lo que lee y solo 27 de cada 100 comprende bien lo que lee, en otras palabras, ingresan a la universidad más estudiantes con bajo nivel de comprensión lectora que los que tienen alto nivel, estos hallazgos se condicen con los de Salas (2012) en México. A este respecto las preguntas que surgen son ¿̇cómo es que jóvenes con bajo nivel de comprensión lectora ingresan a la universidad más exigente de la región? ¿Se mantiene esta situación a lo largo de la carrera?, por lo pronto un estudio de Mendoza (2016) con estudiantes de Psicología, de una universidad particular, en la ciudad de Huancayo ha reportado que no hay diferencias entre los que ingresan a la carrera y los de octavo ciclo, falta saber si esto mismo ocurre en la Universidad Nacional y en otras carreras. Pero żqué significa tener altos o bajos niveles de comprensión lectora? En sentido estricto cuando se habla de comprensión lectora no se hace referencia a la decodificación de las letras escritas, ni únicamente a las palabras 


\section{8}

que forman esas letras. Probablemente, la definición más pertinente la aborda como un proceso en el que, el que lee da un significado a lo que lee con arreglo a los contenidos de su estructura cognitiva Ausubel (1983) y Cooper (1998). La comprensión lectora implica captar no solo palabras sino información, ideas, hasta intenciones del autor del texto. Por eso que, dicha comprensión depende en gran parte de las experiencias del lector de ahí que a la comprensión lectora no se le puede desconocer un inevitable componente subjetivo, en tanto, la comprensión de un texto supone la construcción mental que surge a partir de la interacción del individuo con un texto (Antich, 1986, Collado y García, 1997; Gonzales, 1998 citados por Santiesteban y Velázquez, 2012). Por consiguiente, tener bajos niveles de comprensión lectora implica que, aquellos estudiantes tendrían dificultades para captar las ideas del texto, descubrir las intenciones del autor del mismo y por supuesto, en directa relación con lo apuntado más arriba tener una opinión crítica y fundamentada respecto de todo el texto o una parte de él.

Hasta aquí se ha puesto de manifiesto que el pensamiento crítico, la capacidad para resolver problemas y la comprensión lectora no son requisitos indispensables para el ingreso a la universidad, pero también que las instituciones de secundaria, así como las academias preuniversitarias parecen coincidir en que estas no son cualidades imprescindibles o por lo menos muy necesarias para el estudiante universitario.

Veamos ahora, qué ocurre cuando se separa la muestra en función del sexo. Puede apreciarse que el sub grupo de mujeres tiene más miembros en el nivel alto que los varones (33\% contra $22 \%$ ) y recíprocamente estos tienen más miembros en los niveles bajo y medio que aquellas. Pero estas diferencias no solo son numéricas, sino que alcanzan el nivel de significación, por consiguiente, es legítimo suponer que se encuentran en la población, así puede decirse que al parecer las mujeres aventajan a los varones en la comprensión lectora por lo menos al ingresar a la universidad, falta saber si estas se mantienen a lo largo y al término de la carrera y falta establecer también cuáles son las causas de esas diferencias. Por el momento no podemos atribuírselas a las instituciones educativas ya que los varones y las mujeres de la muestra proceden en la mayoría de los casos de las mismas. Pero además al comparar instituciones estatales con particulares no se encontraron diferencias significativas como tampoco las hubo al comparar a los sub grupos en función del tiempo en que egresaron de la secundaria, de los intentos de ingreso y si realizan o no estudios en paralelo. Esto significa que no porque el estudiante ha ingresado a la universidad después de varios años ha leído más ni alcanzado mayor comprensión que los que acaban de egresar, lo cual, por supuesto 
contradice lo esperado, así como lo deseado. Significa también que el hecho de que no ingresara de primera intención y tuviera que realizar varios intentos para al fin estar dentro de la universidad no condujo al estudiante en general a leer más, ni a desarrollar su comprensión lectora, lo cual indica que probablemente el énfasis en su preparación lo puso en otros aspectos que no tienen que ver con las variables aquí estudiadas. Finalmente significa que esas otras carreras que el subgrupo de estudiantes declara seguir paralelamente tampoco le exigen mayor desarrollo de la comprensión lectora. Por ello es que pueden hacer ambos estudios sin que necesariamente se desarrolle esta capacidad.

Al estudiar la asociación entre la variable pensamiento crítico y capacidad de resolución de problemas, se halló que a diferencia de lo que proponía la hipótesis alterna, el tener mayor nivel de pensamiento crítico no supone necesariamente que el joven estudiante tenga mayor capacidad para la resolución de problemas, lo cual resulta sumamente interesante porque el pensamiento crítico supone también, solucionar problemas solo que en ese caso se trata de analizar la lógica y consistencia de los argumentos. Por consiguiente, no basta con desarrollar con éxito una tabla lógica y puede que ahí radique por lo menos una de las razones de los resultados aquí expuestos. Por supuesto que el pensamiento crítico abarca otras cosas como el análisis de proposiciones, su enjuiciamiento e incluso su contrastación o aplicación con la realidad y esa, la mayor amplitud del pensamiento crítico permitía suponer que quienes tuvieran mayor desarrollo de esta forma de pensamiento tendrían también mayor capacidad de resolución de problemas.

Todo ello conduce a concluir razonablemente que la comprensión lectora, que es una de las capacidades que acusó más déficit en las diversas evaluaciones internacionales y nacionales a las que se sometieron los estudiantes pervanos sigue aún en un marcado déficit y aunque formalmente no debería ser su tarea, la universidad peruana tendrá que sumarse a los esfuerzos para mejorar la comprensión lectora de sus estudiantes.

Por otro lado, al tratar de establecer sí los estudiantes con mayor nivel de desarrollo del pensamiento crítico tenían también mayor desarrollo de la capacidad de resolución de problemas, se encontró que dichas variables no presentan asociación. Lo que significa que, independientemente de que los estudiantes tengan alto, mediano o bajo nivel del desarrollo del pensamiento crítico pueden tener mayor o menor desarrollo en la capacidad de resolución de problemas. Así no sería legítimo tratar de usar como predictor del desempeño del estudiante en la resolución de problemas al nivel 


\section{0}

alcanzado en la prueba de pensamiento crítico. Estos hallazgos resultan sumamente interesantes porque teóricamente hay procesos cognitivos comunes a ambas variables como la comprensión, el análisis, la discriminación, la evaluación, entre otros. No obstante, al parecer, al enfrentar tareas concretas, esta concurrencia de procesos no se manifiesta según lo esperado o tal concurrencia no tiene la relevancia que en teoría debería tener.

Otra es la situación, cuando se analizó la relación entre pensamiento crítico y comprensión lectora. La Chi cuadrado de Pearson, revela que habría asociación entre ambas variables lo que significa que quienes tienen mayor desarrollo del pensamiento crítico tienden a un mayor desarrollo de su comprensión lectora y congruentemente con ello quienes se ubican en el nivel más bajo de la prueba de pensamiento crítico tienden a ubicarse también en el nivel más bajo de la prueba de comprensión lectora. Aquí, sí se puede emplear una variable como predictora de la otra, con cierta legitimidad, aun cuando no se puede afirmar con certeza las relaciones de causa y efecto entre ellas. Será necesario pues, realizar un estudio que ayude a dilucidar este aspecto.

Este hallazgo es hasta cierto punto esperado, ya que, el nivel más alto de la comprensión lectora tal y como se evaluó en este estudio aborda la criticidad del estudiante mientras que el nivel intermedio considerado en la prueba (nivel inferencial) se apoya en la comprensión y el razonamiento respecto de los textos; procesos que también son requeridos al enfrentar la prueba de pensamiento crítico.

Al analizar los procesos cognitivos que intervienen en comprensión lectora; se encontró que en memoria no existen diferencias significativas en el proceso de reconocimiento, pero sí en el de reconstrucción, donde las mujeres son las que obtienen mejores puntuaciones que los varones. En la percepción, solo se encontraron diferencias significativas en el proceso de interpretación a favor de las mujeres. En pensamiento se encontró diferencias significativas en los procesos de juicio y concreción también a favor de mujeres. Sin embargo, hay necesidad de indagar las verdaderas causas de tales diferencias.

\section{Conclusión}

El trabajo ha permitido establecer que los ingresantes a la Universidad Nacional del Centro del Perú, pueden hacerlo aun presentando un bajo nivel de desarrollo del pensamiento crítico; por consiguiente, la universidad no estaría evaluando esta 
competencia como requisito para el ingreso. Que las mujeres tienen mayor desarrollo de esta forma de pensamiento que los varones, lo cual no puede ser achacado a la educación formal; que el número de intentos para ingresar a la universidad, los años que median entre el ingreso a la universidad y el egreso del colegio, así como el hecho de estudiar otra carrera paralelamente son irrelevantes en el desarrollo del pensamiento crítico.

Que más de la mitad de jóvenes que ingresan a la universidad tienen escaso desarrollo de la capacidad de resolución de problemas, lo cual sugiere que la universidad pública no está poniendo como requisito para el ingreso esta capacidad; que en esto no se diferencian varones con mujeres y que el tener el mayor o menor desarrollo del pensamiento crítico no se relaciona con el nivel de desarrollo de la capacidad de resolución de problemas. Pero esto, obliga a seguir investigando, ya que hipotéticamente ambas variables deberían estar relacionadas en tanto son expresiones del pensamiento. La comprensión lectora es una competencia que muchos ingresantes tienen poco desarrollada; que las mujeres la tienen más desarrollada que los varones. Y que esta sí está asociada con el pensamiento crítico por consiguiente la primera puede funcionar como predictora de la otra, lo cual se condice con los constructos teóricos subyacentes.

\section{Referencias}

Alarcón, R. (1991). Métodos y Diseños de Investigación del Comportamiento. Lima. Fondo Editorial.

Alejo, L. (2017). El pensamiento crítico en estudiantes del grado de maestro/a en educación primaria desde la didáctica de las ciencias sociales, [tesis doctoral]. https://riuma.uma.es/xmlui/bitstream/handle/10630/15997/

TD_ALEJO_LOZANO_Laura.pdf? sequence $=1$

Alonso, J., Coronel, C. y Guevara, J. (2016). "Comprensión lectora en resolución de problemas matemáticos en alumnos universitarios", [tesis de licenciatura, Universidad Nacional de Trujillo]. http://dspace.unitru. edu.pe/bitstream/handle/UNITRU/5125/ALONZO\%20CRUZCORONEL\%2OROMEROGUEVARA\%20GUZMAN.pdf? sequence $=1$ \&isAllowed=y

Altuve, G., J. G. (2010). El pensamiento crítico y su inserción en la educación superior. Actualidad Contable Faces, 13 (20) 5-18. http://www. redalyc.org/comocitar. oa? id $=25715828002$ 


\section{2}

Ausubel, D. (1983). Psicología educativa: un punto de vista cognoscitivo. $2^{\circ}$ Ed, Trillas. México.

BBC News Mundo (3 de diciembre de 2019). Pruebas PISA: qué países tienen la mejor educación del mundo (y qué lugar ocupa América Latina en la clasificación). Recuperado de https://www.bbc.com/mundo/noticias-internacional-50643441

Bringas, R.; Caro, B. y Trigozo, J. (2018) Propuesta de programación del área de Historia, Geografía y Economía para desarrollar el pensamiento crítico en estudiantes del cuarto año de educación secundaria de una institución educativa privada de Puente Piedra - Lima, [tesis de licenciatura, Universidad Marcelino Champagnat]. Repositorio Institucional UMCH. http://repositorio.umch.edu.pe/handle/UMCH/463

Bono (De), E. (2014). El pensamiento lateral: Manual de creatividad. Editorial Paidos. México.

Campos, A. (2007). Pensamiento crítico. Técnicas para su desarrollo. Editorial Cooperativa. Bogotá.

Carretero, J. (1995). Lecturas de psicología: Razonamiento, solución de problemas y desarrollo cognitivo. Editorial Alianza. España.

Cooper, D. (1998). Cómo mejorar la comprensión lectora. Madrid. España.

Cuñachi, G. y Leyva, G. (2018). Comprensión lectora y el aprendizaje en el área de Comunicación Integral en los estudiantes de Educación Básica Alternativa de las instituciones educativas del distrito de Chaclacayo, UGEL06, Ate vitarte año 2015, [tesis de licenciatura, Universidad Nacional de Educación Enrique Guzmán y Valle] Repositorio Institucional UNEEGV. http://repositorio.une. edu.pe/handle/UNE/1335

Cruz, I. (2015) Uso de las redes sociales para la resolución de problemas matemáticos en estudiantes no universitarios, [tesis doctoral, Universidad de Sevilla] Base de datos US. https://idus.us.es/xmlui/handle/11441/34121

ECE: Menos del $20 \%$ de escolares de $2^{\circ}$ de secundaria entiende lo que lee ( 7 abril, 2019). La República. https://larepublica.pe/sociedad/1445612-ece-20-escolares2deg-secundaria-entiende-lee/

Escalante, S. (2015) Método Pólya en la Resolución de Problemas Matemáticos, [tesis de licenciatura, Universidad Rafael Landívar]. http://recursosbiblio.url.edu.gt/tesisjcem/2015/05/86/Escalante-Silvia.pdf 
Fátima, V. (18 de junio de 2012). Alfa de Cronbach. Obtenido de Slide share: https://es.slideshare.net/ViancaFatima_1990/18-septiembre-2007-alfa-decrombach-13371250? qid=75eee538-a415-4f63-bdbacabba6b6d $12 f \& v=\& b=\&$ from_search $=5$

Faccione, P. (2007). Pensamiento Crítico: ¿¿Qué es y por qué es importante? (I. Assessment, Ed.) http://www.insightassessment.com

Garret, R. M. (1988) Resolución de problemas y creatividad: implicaciones para el currículo de Ciencias. Barcelona

Gonzales, C., R. (2016). Pensamiento crítico y liderazgo en docentes de primaria de instituciones estatales de la región Junín, [tesis de maestría Universidad Nacional del Centro del Perú]. Repositorio institucional UNCP. http://repositorio.uncp.edu.pe/handle/UNCP/4301

Guevara, F. (2016) El Pensamiento crítico y su relación con el desempeño docente en el décimo ciclo de pregrado, de Educación de la Universidad Nacional Mayor de San Marcos, [tesis de maestria, Universidad Nacional Mayor de San Marcos ]. Repositorio Institucional cibertesis UNMSM. https://hdl.handle.net/20.500.12672/6399

Gutiérrez, J. (2012). Estrategias de enseñanza y resolución de problemas matemáticos según la percepción de estudiantes del cuarto grado de primaria de una institución educativa - ventanilla. [Tesis magistral, Universidad San Ignacio de Loyola]. http://repositorio.usil. edu.pe/bitstream/123456789/1201/1/2012_Guti\%C3\%A9rrez_Estrategias\%20de\%20 ense\%C3\%B 1 anza\%20y\%20resoluci\%C3\%B3n\%20de\%20problemas\%20matem\%C3 \%A 1 ticos\%20seg\%C3\%BAn\%20la\%20percepci\%C3\%B3n\%20de\%20estudiantes\%20 del\%20cuarto\%20grado\%20de\%20primaria.pdf

Javier, L. (2003). Estabilidad de la atención y solución de problemas racionales en estudiantes de educación de las universidades estatales de Huancayo y Huancavelica.

Luria, A. (1974). El cerebro en acción. Barcelona: Fontanella.

Luria A. (1984). Exploración de las funciones corticales superiores. Editorial: Martínez Roca.

Luria, A. (2013) Las funciones corticales superiores del hombre. Editorial Fontamara. México 


\section{4}

Macedo, A. (2018). Pensamiento crítico y rendimiento académico en los ingresantes del curso de Estadística I en la Facultad de Ingeniería Económica, Estadística y Ciencias Sociales. Universidad Nacional de Ingeniería - 2017, [tesis de maestría, Universidad Peruana Cayetano Heredia] Repositorio UPCH. http://repositorio.upch.edu.pe/ handle/upch/1541

Manuale, M. (2007). Estrategias para la comprensión: construir una didáctica para la educación superior. Argentina: UNL

Matalinares, M. (1999). Incremento de la eficacia para la solución de problemas en alumnos de quinto de secundaria, [tesis de maestría, Universidad Nacional Mayor de San Marcos].

Mejía, A. y Loangov, M. (2014). Resolución de matemáticos problemas para fortalecer el pensamiento numérico en estudiantes del grado séptimo de la Institución Educativa Adventista del Municipio de Puerto Tejada Cauca, [tesis de licenciatura, Universidad Católica de Manizales]. http://repositorio.ucm.edu.co:8080/jspui/bitstream/handle/10839/848/Aida\%20C onsuelo\%20Mejia\%20Viafara.pdf? sequence=1 \&isAllowed=y

Mendoza, J. L. (2016) La investigación y el desarrollo de pensamiento crítico en estudiantes universitarios, [tesis doctoral, Universidad de Málaga]. http://hdl.handle.net/10630/11883

Ministerio de Educación (2016). Cuanto aprenden los alumnos. Resultados ECE 2016. http://umc.minedu.gob.pe/wp-content/uploads/2017/04/ResultadosNacionales-2016.pdf

Pinedo, M. y Cerrón, A. (julio, 2015). Pensamiento crítico y rendimiento académico de estudiantes de la Facultad de Educación de la Universidad Nacional del Centro del Perú. Horizonte de la ciencia. 5 (8) 105-110.

Pino, J. (2012) Concepciones y prácticas de los estudiantes de Pedagogía Media en Matemáticas con respecto a la Resolución de Problemas y, diseño e implementación de un curso para aprender a enseñar a resolver problemas, [tesis doctoral, Universidad de Extremadura] http://dehesa.unex.es/bitstream/handle/10662/568/TDUEX_2013_Pino_Ceballos.p df? sequence $=1$ \&isAllowed $=y$

Polya, G. (1965). Cómo plantear y resolver problemas. Editorial Trillas. México.

Rugarcia, G. (1999) La investigación y el desarrollo de pensamiento crítico en estudiantes universitarios, [†esis doctoral, Universidad de Málaga]. 
https://riuma.uma.es/xmlui/bitstream/handle/10630/1 1883/TD_MENDOZA_GUERR ERO_Pedro_Luis.pdf? sequence=1

Sabucedo, J., M. y Morales, J., F. (2015). Psicología social. Editorial Médica Panamericana, Málaga.

Salas, P. (2012) Desarrollo de la comprensión lectora en los estudiantes de tercer semestre del nivel medio superior de la Universidad Autónoma de Nuevo León, (tesis de maestría, Universidad Autónoma de Nuevo León). Repositorio institucional UANL. http://eprints.uanl.mx/3230/1/1080256466.pdf

Saiz, C., \& Rivas, S. (8 y 11 , enero, 2008). Intervenir para transferir en pensamiento crítico [Presentación de la conferencia] Conferencia internacional: Lógica, Argumentación y Pensamiento Crítico. Santiago de Chile, Chile. http://www.pensamientocritico.com/archivos/intervensa izrivas.pdf

Sánchez, H., y Reyes, C. (2016). Metodología y diseños en la investigación científica (2a ed.). Lima: Visión Universitaria.

Santiesteban, E. y Velázquez, K. M. (2012). La comprensión lectora desde una concepción didáctico cognitiva. Didáctica y educación. 3 (1), 103-110. https://dialnet.unirioja.es/servlet/articulo? codigo $=4228654$

Solé, I. (2001). Estrategias de Lectura:12 va. Edición. Barcelona: ICE de la universitat de Barcelona y EditoriaL Graó.

Schoenfeld, A.H. (1985). Ideas y tendencias en la resolución de problemas, en MEC, La enseñanza de la matemática a debate. (Subdirección General del Perfeccionamiento del Profesorado: Madrid), pp. 25-30

Vela, N. (2015) Hábitos de estudio y Comprensión Lectora en estudiantes del $2^{\circ}$ grado de primaria, I.E.P.S.M. n 60050 República de Venezuela, lquitos - 2014, [tesis de licenciatura, Universidad Nacional de la Amazonia Peruana] UNAPhttp://repositorio.unapiquitos.edu.pe/bitstream/handle/UNAP/4581/Nury_Tesis_Tit ulo_2015.pdf? sequence=1 \&isAllowed=y

Yarlequé, L. (1994). Psicología Evolutiva y Pedagógica. Huancayo: Universidad Nacional del Centro del Perú

Yarlequé, L. Javier, L. Monroe, J. y Nuñez, E. (2007). Investigación en Educación y Ciencias Sociales. Huancayo.

Yarlequé, L., Javier, L., Nuñez, E., Navarro, L., Cerrón, A. y Monroe, J. (julio, 2012). Comprensión lectora en los docentes de Educación Básica de la Región Junín. 


\section{6}

Revista de Investigación en Psicología.15 (1) 83-91.

https://doi.org/10.15381/rinvp.v15i1.3666

Yarlequé, L. y Moya, N. (2009). Logro de competencias en la educación. Huancayo.

Yarlequé, L., Nuñez, E. y Navarro, L. (junio de 2012). Programa LEFI y lectura eficiente en docentes de la especialización en comunicación y matemática de II y III ciclo de la Región Junín. Horizonte de la Ciencia (02) , 47-55.

Zárate, A. (2019). Autoeficacia hacia la lectura, comprensión de lectura y rendimiento académico en estudiantes de primer ingreso de una Universidad Privada de Lima, [tesis de maestría]. Repositorio institucional. http://repositorio.usmp.edu.pe/handle/usmp/ 4587 